\title{
FRENET THEORY OF SURFACES
}

\author{
TIMO RAVASKA
}

\section{Introduction}

In the present paper we consider smooth mappings $y: U \rightarrow F, U \subset E, E$ being a finite-dimensional real linear space and $F$ euclidean. Since the image of $U$ is a surface in $F$, this forms a part of surface theory.

Our starting point is the paper [6] by Rikkonen, on p. 26 of which he formulates the embedding theorem. We wish to consider this theorem more profoundly, providing on p. 159 below our own version, which yields more exact information on the dimensions of the tangent spaces of the surface $M$.

An embedding theorem is given also by Guggenheimer [2, p. 232], using terms of differential forms and exterior calculus. This, however, does not give any information of the dimensions of the tangent spaces of the surface.

We also refer to [7], Volumes 4 and 5.

We are also able here, on p. 162 to establish a theorem concerning the degeneration of a surface, treating this as analogous to the degeneration of a curve [5, p. 190]. The proof, however, is entirely different.

Having defined the "curvatures" of a surface on p. 155, we shall also go on to consider surfaces with constant curvatures.

Our method is based on the absolute analysis of several variables from [5], and employs linear operators in a fashion analogous to that of curve theory [5, pp. 185-193]. This enables us to avoid the use of Cartesian product spaces as introduced in [6, p. 23], gives a more natural setting for the theory, and leads to more detailed results.

\section{Notations and conventions}

Let $E$ be a $k$-dimensional vector space over $\boldsymbol{R}(k<\infty)$ and let $S=\left\{x_{1}, x_{2}, \ldots, x_{n}\right\}$ be a set of vectors in $E$. Denote by $\operatorname{sp}\left\{x_{1}, \ldots, x_{n}\right\}=\operatorname{sp}(S)$ the linear subspace of $E$ spanned by $S$.

Let $y^{(p)}$ be a $p$-linear function $\times_{1}^{p} E \rightarrow F$, where $F$ is a real vector space. We denote the linear closure of the range of $y^{(p)}$ by

$$
\operatorname{Im}\left(y^{(p)}\right)=\operatorname{sp}\left\{y^{(p)} x_{1} \ldots x_{p} \mid x_{i} \in E, i=1, \ldots, p\right\} .
$$


It is enough to take a basis $\left\{a_{j}\right\}_{1}^{k}$ of $E$ in (1) to obtain $\operatorname{Im}\left(y^{(p)}\right)$ :

$$
\operatorname{Im}\left(y^{(p)}\right)=\operatorname{sp}\left\{y^{(p)} a_{i_{1}} a_{i_{2}} \ldots a_{i_{p}} \mid a_{i_{j}} \in\left\{a_{q}\right\}_{1}^{k}, j=1, \ldots, p\right\} .
$$

Since we shall consider symmetric multilinear functions, we will also introduce the following notations.

Let $S_{n, k}$ be the totality of nondecreasing sequences of positive integers of length $n$ chosen from $\{1,2, \ldots, k\}, n=0,1,2, \ldots$. In particular, $S_{0, k}=\emptyset$, and $S_{1, k}$ can be identified with $\{1,2, \ldots, k\}$. The elements of $S_{n, k}$ are denoted by $\bar{i}, \bar{j}, \ldots$.

We fix a complete ordering $<$ for the elements of $S_{n, k}, n=1,2, \ldots$, i.e. for $\bar{i} \in S_{m, k}, \bar{j} \in S_{n, k}$, either $\bar{i}<\bar{j}, \bar{i}=\bar{j}$ or else $\bar{i}>\bar{j}$. In particular, if $m<n$, then $\tilde{i}<j$. We denote $S_{n, k}$ with this ordering by $O_{n, k}, n=0,1,2, \ldots$.

The values of a symmetrical multilinear function $y^{(n)}$ for a sequence $\left(a_{i_{1}}, a_{i_{2}}, \ldots, a_{i_{n}}\right)$ can be expressed as

$$
y^{(n)} a_{i_{1}} \ldots a_{i_{n}}=y^{(n)} a_{q_{1}} a_{q_{2}} \ldots a_{q_{n}}, \quad\left(q_{1}, \ldots, q_{n}\right) \in O_{n, k},
$$

by virtue of the symmetry.

\section{Tangent spaces}

Let $E$ be as above and let $F$ be an $m$-dimensional $(k<m<\infty)$ euclidean vector space. Let $M$ be a $k$-dimensional $C^{\infty}$ surface in $F$ parametrized by an open region $U$ of the parameter space $E$. Hence we have for $M$ a parameter representation

$$
y=y(x), \quad x \in U \subset E,
$$

where $y: U \rightarrow F$ is a $C^{\infty}$ mapping and its derivative $y^{\prime}(x): E \rightarrow F$ is a regular linear mapping for all $x \in U[5$, p. 181].

Given any $x \in U$, the $q$-th derivative $y^{(q)}(x)$ is a symmetric $q$-linear function $\times_{1}^{q} E \rightarrow F, q=1,2, \ldots$.

The $n$-th tangent space of the surface $M$ at $y \in M$ is

$$
T_{y}^{n}(M)=\sum_{q=1}^{n} \operatorname{Im}\left(y^{(q)}(x)\right), \quad n=1,2, \ldots
$$

These sums of linear subspaces are not necessarily direct sums, since $\operatorname{Im}\left(y^{(i)}(x)\right) \cap \operatorname{Im}\left(y^{(j)}(x)\right)$ may be $\neq\{0\}$ for $i \neq j$. Let us assume that there exists an integer $p$ such that $T_{y}^{p}(M)=F$ and that $\operatorname{dim} T_{y}^{n}(M)=\sum_{i=1}^{n} m_{i}(n=1, \ldots, p)$ is independent of the point $y \in M$. Thus we say that the surface $M$ is of type $\left(m_{1}, m_{2}, \ldots, m_{p}\right)$.

We may now construct a basis for $T_{y}^{n}(M)$ as follows.

Let $\left\{a_{1}, a_{2}, \ldots, a_{k}\right\}$ be a basis of $E$. Since the subspace $\operatorname{Im}\left(y^{(n)}(x)\right)$ is spanned by the elements

$$
y^{(n)}(x) a_{i_{1}} a_{i_{2}} \ldots a_{i_{n}}, \quad\left(i_{1}, \ldots, i_{n}\right) \in O_{n, k}, \quad n=1,2, \ldots, p, \quad x \in U,
$$

we can use these vectors of $F$ in our construction. 
Since $y^{\prime}(x) a_{i}, i=1, \ldots, k$ are linearly independent vectors of $F$, they form a basis for $T_{y}^{1}(M)$. We then consider the vectors $y^{\prime \prime}(x) a_{i} a_{j},(i, j) \in O_{2, k}$, arranged according to the ordering of $O_{2, k}$. We proceed in this sequence dropping out any element which is linearly dependent on any elements preceding it (including elements of $\left.T_{y}^{1}(M)\right)$.

The remaining elements $y^{\prime \prime} a_{i} a_{j}$ define a subset $o_{2, k}$ of $O_{2, k}$.

Hence we obtain a basis for $T_{y}^{2}(M)$, namely,

$$
\left\{y^{\prime}(x) a_{i} \mid i=1, \ldots, k\right\} \cup\left\{y^{\prime \prime}(x) a_{i} a_{j} \mid(i, j) \in o_{2, k}\right\} .
$$

Continuation of this procedure yields

Lemma 1. Given a basis $\left\{a_{i}\right\}_{1}^{k}$ of the parameter space $E$, there exist subsets $o_{q, k} \subset O_{q, k}$ with $m_{q}$ elements, $q=1,2, \ldots, n$, such that

$$
\bigcup_{q=1}^{n}\left\{y^{(q)}(x) a_{i_{1}} \ldots a_{i_{q}} \mid\left(i_{1}, \ldots, i_{q}\right) \in o_{q, k}\right\}
$$

is a basis for the $n$-th tangent space $T_{y}^{n}(M), n=1,2, \ldots, p$. Here $m_{q}=\operatorname{dim} N_{y}^{q}(M)$, $N_{y}^{q}(M)=T_{y}^{q}(M)^{\perp} \cap T_{y}^{q+1}(M)$ being the $q$-th normal space, $q=1, \ldots, p-1, m_{1}=k=$ $\operatorname{dim} T_{y}^{1}(M)$ (see [6, p. 8]).

Suppose that the index sets $o_{n, k}, n=0,1, \ldots, p$ are independent of $x \in U$. It also follows that the type of the surface is independent of $x \in U$.

\section{Accompanying orthonormal frame and Frenet formulas}

We take $n=p$ in (6) obtaining a basis of $F$. Then we orthonormalize this sequence by means of the Schmidt orthonormalization procedure, the result

$$
e_{\bar{i}}(x), \quad \bar{i} \in o_{n, k}, \quad n=1,2, \ldots, p, \quad x \in U
$$

being the accompanying orthonormal frame of the surface $M$ at $y=y(x), x \in U$.

3.1. Orthonormalization in $T_{y}^{1}(M)$. We obtain in $T_{y}^{1}(M)$ the formulas

$$
y^{\prime}(x) a_{j}=\sum_{i=1}^{j} \lambda_{j}^{i}(x) e_{i}(x), \quad j=1,2, \ldots, k, \quad x \in U
$$

with $C^{\infty}$ coefficients

and

$$
\lambda_{j}^{i}(x)=\left(y^{\prime}(x) a_{j} \mid e_{i}(x)\right), \quad i=1,2, \ldots, j-1
$$

$$
\lambda_{j}^{j}(x)=\left|y^{\prime}(x) a_{j}-\sum_{i=1}^{j-1} \lambda_{j}^{i}(x) e_{i}(x)\right|, \quad j=1, \ldots, k,
$$

$\left\{a_{i}\right\}_{1}^{k}$ being a basis of the parameter space $E$. 
In view of the fact that the first fundamental form $G(x): E \times E \rightarrow \boldsymbol{R}$ (see [5, p. 193]) of $M$ is defined by

$$
G(x) a b=(a \mid b)_{x}=\left(y^{\prime}(x) a \mid y^{\prime}(x) b\right), \quad a, b \in E, \quad x \in U,
$$

the formulas (7) imply

$$
g_{i j}(x)=G(x) a_{i} a_{j}=\sum_{n=1}^{\min \{i, j\}} \lambda_{i}^{n}(x) \lambda_{j}^{n}(x), \quad i, j=1,2, \ldots, k .
$$

Conversely, given $\left\{g_{i j}(x)\right\}_{1}^{k}$, one obtains the coefficients $\left\{\lambda_{j}^{i}(x)\right\}$ recursively from

$$
\left\{\begin{array}{l}
\lambda_{j}^{1}(x)=g_{1 j}(x)\left(g_{11}(x)\right)^{-1 / 2}, \quad j=1,2, \ldots, k \\
\lambda_{j}^{i}(x)=\frac{g_{i j}(x)-\sum_{n=1}^{i-1} \lambda_{i}^{n}(x) \lambda_{j}^{n}(x)}{\left[g_{i l}(x)-\sum_{n=1}^{i-1}\left(\lambda_{i}^{n}(x)\right)^{2}\right]^{1 / 2}}, \quad 2 \leqq i \leqq j \leqq k .
\end{array}\right.
$$

3.2. General orthonormalization formulas. The general orthonormalization formulas are

$$
y^{(q)}(x) a_{j_{1}} a_{j_{2}} \ldots a_{j_{q}}=\sum_{n=1}^{q} \sum_{\substack{i \in o_{n, k} \\ i \leqq j}} \lambda_{\bar{j}}^{\bar{i}}(x) e_{\bar{i}}(x)
$$

$\left(j_{1}, \ldots, j_{q}\right)=\bar{j} \in o_{q, k} ; x \in U \subset E$, with $C^{\infty}$ coefficients

and

$$
\lambda_{\bar{j}}^{\bar{i}}(x)=\left(y^{(q)} a_{j_{1}} \ldots a_{j_{q}} \mid e_{\bar{i}}\right), \quad \bar{i}<\bar{j},\left\{\begin{array}{l}
\bar{i} \in o_{n, k}, \quad 1 \leqq n \leqq q, \\
\bar{j} \in o_{q, k}
\end{array}\right.
$$

where $p_{\bar{j}}$ is the projection of $y^{(q)}(x) a_{j_{1}} \ldots a_{j_{q}}$ on the linear subspace of $F$ spanned by those $e_{\bar{i}}(x)$ for which $\bar{i}<\bar{j}$.

Remark. The formula (12) applies to the elements of the basis from (6). Since $\left\{e_{\bar{i}}(x)\right\}$ is also a basis, all the derivatives of $y$ can be expressed by means of this basis, and we may hereby generalize the formula (12) for all the vectors $y^{(q)} a_{j_{1}} \ldots a_{j_{q}},\left(j_{1}, \ldots, j_{q}\right) \in O_{q, k}, q=1,2, \ldots, p$.

Lemma 2. The functions $e_{\bar{i}}$ as functions of $x \in U$ are twice differentiable with a symmetric second derivative.

3.3. Frenet formulas. The construction of the accompanying orthonormal frame shows that for $\bar{i} \in o_{n, k}, x \in U$,

$$
e_{\bar{i}}(x) \in T_{y}^{n}(M) \cap T_{y}^{n-1}(M)^{\perp}=N_{y}^{n-1}(M), \quad n=2, \ldots, p .
$$

Let us fix an $a \in E$ and differentiate $e_{\bar{i}}(x)$ with respect to $a$. Then $e_{\bar{i}}^{\prime}(x) a \in T_{y}^{n+1}(M)$ for $\tilde{i} \in o_{n, k}, n=1, \ldots, p-1$, and hence

$$
\left(e_{i}^{\prime}(x) a \mid e_{j}(x)\right)=0 \quad \text { for } \quad \bar{j} \in o_{q, k}, \quad q>n+1
$$


We now differentiate the relation $\left(e_{\tilde{i}}(x) \mid e_{j}(x)\right)=0$ with respect to $a \in E$ to obtain $\left(e_{i}(x) \mid e_{\bar{j}}^{\prime}(x) a\right)=0$ for $\bar{j} \in o_{q, k}, \bar{i} \in o_{n, k}, q>n+1$. With $\bar{i}$ and $\bar{j}$ interchanged, the symmetry of the scalar product yields

$$
\left(e_{i}^{\prime}(x) a \mid e_{j}(x)\right)=0 \text { for }\left\{\begin{array}{l}
\bar{i} \in o_{n, k} \\
\bar{j} \in o_{q, k}
\end{array}, \quad q>n+1 \quad \text { or } \quad q<n-1 .\right.
$$

Then, for $a \in E$ (cf. [7, Vol. 4, p. 297]),

and we have

$$
e_{i}^{\prime}(x) a \in \begin{cases}T_{y}^{1}(M) \oplus N_{y}^{1}(M) & \text { for } n=1 \\ N_{y}^{n-2}(M) \oplus N_{y}^{n-1}(M) \oplus N_{y}^{n}(M), & 2 \leqq n \leqq p-1 \\ N_{y}^{p-2}(M) \oplus N_{y}^{p-1}(M) & \text { for } n=p,\end{cases}
$$

Lemma 3. There exist real coefficients $x_{j}^{\bar{i}}(x, a), x \in U \subset E, a \in E$, indexed in such $a$ way that if $\bar{j} \in o_{n, k}$, then $\bar{i} \in o_{n-1, k}, o_{n, k}$ or $o_{n+1, k}$ for $n=1,2, \ldots, p-1$ $\left(o_{0, k}=\emptyset\right)$, and if $\bar{j} \in o_{p, k}$, then $\bar{i} \in o_{p-1, k}$ or $o_{p, k}$, such that

$$
\begin{aligned}
e_{j}^{\prime}(x) a & =\sum_{i \in o_{n-1, k}} x_{\bar{j}}^{\bar{i}}(x, a) e_{i}(x)+\sum_{i \in o_{n, k}} x_{\bar{j}}^{\bar{i}}(x, a) e_{\bar{i}}(x) \\
& +\sum_{\bar{i} \in o_{n+1, k}} x_{\bar{j}}^{\bar{i}}(x, a) e_{\bar{i}}(x), \quad \bar{j} \in o_{n, k}, \quad n=1,2, \ldots, p,
\end{aligned}
$$

(for $n=1$, discard the first of these sums, and for $n=p$ the last).

For each $x \in U \subset E$ the function $x_{\bar{j}}^{\bar{i}}(x, a)$ is linear in $a \in E$ and for each $a \in E$ the functions $x_{\bar{j}}^{\bar{i}}(x, a)$ are $C^{1}$ functions of $x$.

We call the formulas (15) the Frenet formulas of $M$ and the numbers $x_{j}^{i}(x, a) \in \boldsymbol{R}$ the curvatures of $M$ with respect to the vector $a \in E$, and denote $x_{\tilde{j}}^{\bar{i}}(x, a)=x_{\bar{j}}^{\bar{i}}(x) a$.

Remark. The formulas (15) are formally the same as (1.50) in [6, p. 21].

See also [7, Vol. 4, p. 250].

For each $x \in U, a \in E$ we define the Frenet operator $A(x, a)$ as the linear operator $A(x, a): F \rightarrow F$ satisfying

$$
A(x, a) e_{\bar{j}}(x)=e_{\bar{j}}^{\prime}(x) a, \quad \bar{j} \in o_{n, k}, \quad n=1,2, \ldots, p
$$

and being thus completely determined by means of its values for the basis $\left\{e_{j}(x)\right\}$ of $F$.

It is clear from (16) that $A(x, a)$ as a function of $a$ is a linear map from $E$ to the space $L(F ; F)$ of linear mappings $F \rightarrow F$. We denote $A(x, a)=A(x) a$. For each $x \in U, A(x)$ is then a bilinear operator $E \times F \rightarrow F$.

The orthonormality of the basis $\left\{e_{i}(x)\right\}$ implies that for each $x \in U, a \in E$ the operator $A(x) a: F \rightarrow F$ is skew-symmetric:

$$
[A(x) a]^{*}=-A(x) a .
$$


Hence for $n=2,3, \ldots, p-1$,

$$
\varkappa_{\bar{j}}^{\bar{i}}(x) a=-\chi_{\bar{i}}^{\bar{j}}(x) a, \quad \bar{j} \in o_{n, k}, \quad \bar{i} \in o_{n-1, k}, o_{n, k} \quad \text { or } \quad o_{n+1, k}
$$

and, in particular, $\chi_{i}^{\bar{i}}(x) a \equiv 0, x \in U, a \in E$. The functionals $\left\{x_{j}^{\bar{i}}(x)\right\}(\bar{i}>\bar{j})$ are subsequently referred to as the curvatures of $M$.

We call the matrix of the Frenet operator $A(x) a$ with respect to the basis $\left\{e_{i}(x)\right\}$ the Frenet matrix. This can be expressed in a partitioned form as

$$
[A(x) a]=\left(\begin{array}{cccccc}
A_{11} & -A_{21}^{*} & 0 & \ldots & 0 & 0 \\
A_{21} & A_{22} & -A_{32}^{*} & \ldots & 0 & 0 \\
0 & A_{32} & A_{33} & & & \\
\vdots & \vdots & & \ddots & & \vdots \\
0 & 0 & & & A_{p-1, p-1} & -A_{p, p-1}^{*} \\
0 & 0 & \ldots & & A_{p, p-1} & A_{p p}
\end{array}\right),
$$

the asterisk * denoting the transpose of a matrix.

Assuming that the elements of $o_{n, k}$ are in the proper order $(\bar{i}, \bar{j}, \ldots, \bar{q}, \bar{r})$, the diagonal submatrices $A_{n n}, n=1, \ldots, p$ from (19) are antisymmetrical $m_{n} \times m_{n}$ matrices

$$
A_{n n}=\left(\begin{array}{ccccc}
0 & -\chi_{\bar{i}}^{\bar{j}}(x) a & \ldots & -\chi_{\overline{\bar{q}}}^{\bar{q}}(x) a & -\chi_{\bar{i}}^{\bar{r}}(x) a \\
\chi_{\bar{i}}^{\bar{j}}(x) a & 0 & & -\chi_{\bar{j}}^{\bar{q}}(x) a & -\chi_{\bar{j}}^{\bar{r}}(x) a \\
\vdots & & \ddots & \vdots & \vdots \\
\chi_{\bar{q}}^{\bar{q}}(x) a & \chi_{\overline{\bar{q}}}^{\bar{q}}(x) a & \ldots & 0 & -\chi_{\bar{q}}^{\bar{r}}(x) a \\
\chi_{\bar{i}}^{\bar{r}}(x) a & \chi_{\bar{j}}^{\bar{r}}(x) a & \ldots & \chi_{\bar{q}}^{\bar{r}}(x) a & 0
\end{array}\right), \quad n=1, \ldots, p .
$$

Moreover, if the elements of $o_{n+1, k}$ are allowed to be $(\bar{k}, \bar{l}, \ldots, \bar{s}, \bar{t})$, the submatrices $A_{n+1, n}$ from (19) are $m_{n+1} \times m_{n}$-matrices of the form

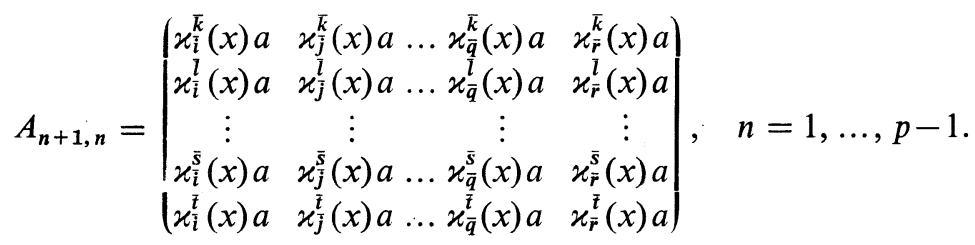

Remark. It is easily seen that the curvatures remain the same if the surface is transformed by means of an orthogonal transformation of $F$.

Lemma 2 and (16) together give

Lemma 4. The bilinear operator $A(x): E \times F \rightarrow F$ satisfies

$$
\bigwedge_{a, b}\left[A^{\prime}(x) a b-A(x) a A(x) b\right]=0 \quad(a, b \in E, x \in U) .
$$

Remark. The formula (20) is not very useful because the operator $A(x)$ is defined with respect to a "moving" basis. 


\section{Linear operators associated with the surface}

Let $x_{0} \in U$ be a fixed point. Define an orthogonal operator $T(x): F \rightarrow F$ by

$$
T(x) e_{\bar{i}}\left(x_{0}\right)=e_{\bar{i}}(x), \quad x \in U, \quad \bar{i} \in o_{n, k}, \quad n=1, \ldots, p .
$$

Then $T$ as a function of $x$ is $C^{2}$ satisfying

$$
T^{\prime}(x) a=A(x) a T(x), \quad a \in E, \quad x \in U, \quad T\left(x_{0}\right)=I .
$$

Clearly (20) is the integrability condition for the total differential equation (22) (cf. [5, p. 164]).

In view of the Remark following (20), we consider a bilinear operator $B(x): E \times F \rightarrow F$ defined by the property that it possesses the matrix (19) with respect to the fixed basis $\left\{e_{\bar{i}}\left(x_{0}\right)\right\}$ of $F$. Then the linear operator $B(x) a: F \rightarrow F$ is skewsymmetric, and we have

Lemma 5. The bilinear operator $B(x): E \times F \rightarrow F$ satisfies for all $a, b \in E, x \in U$

$$
[B(x) a]^{*}=-B(x) a
$$

$$
T^{\prime}(x) a=T(x) B(x) a, \quad x \in U, \quad a \in E, \quad T\left(x_{0}\right)=I .
$$

this being the integrability condition for the total differential equation (24).

Remark. The formula (25) corresponds to the integrability conditions $(1.51-54)$ in [6], p. 21, i.e. the Gauss, Codazzi and Ricci equations (see also [7]).

Consider now the orthogonal projection operators $P_{n}(x): F \rightarrow N_{y}^{n-1}(M)$, $n=1,2, \ldots, p\left(N_{y}^{0}(M)=T_{y}^{1}(M)\right)$; cf. [6, p. 9]. These can be defined by

$$
P_{n}(x) e_{i}(x)=\left\{\begin{array}{ll}
e_{i}(x) & \text { if } \quad \bar{i} \in o_{n, k} \\
0 & \text { if } \quad \bar{i} \in o_{q, k}
\end{array} \text { for } q \neq n, \quad q, n=1, \ldots, p .\right.
$$

By reference to (21), an easy computation yields

$$
P_{n}(x)=T(x) P_{n}\left(x_{0}\right) T(x)^{-1}, \quad n=1,2, \ldots, p .
$$

Differentiating (26) and using (16) we obtain

$$
P_{n}^{\prime}(x) a=\left[A(x) a, P_{n}(x)\right], \quad a \in E, \quad x \in U, \quad n=1, \ldots, p,
$$

where the brackets denote the commutator of two linear operators.

A connection with the Rikkonen formulas [6, p. 14], is obtained from the following 
Proposition 1. The orthogonal projections $P_{n}(x), x \in U, n=1,2, \ldots, p$ satisfy for $a \in E$

$$
P_{n}^{\prime}(x) a P_{n}(x)=\left(I-P_{n}(x)\right) A(x) a, \quad n=1, \ldots, p .
$$

Moreover,

$$
P_{n}^{\prime}(x) a P_{n+1}(x)=-P_{n}(x) A(x) a P_{n+1}(x), \quad n=1, \ldots, p-1
$$

\section{Relations between curvatures and orthonormalization coefficients}

Let us consider more closely the orthonormalization coefficients $\left\{\lambda_{j}^{i}\right\}$ in (12), in the light of the curvatures $\left\{x_{\bar{j}}^{\bar{i}}(x)\right\}$ from (15).

Our starting point in the first tangent space $T_{y}^{1}(M)$ is slightly different from the one used in [6, p. 19], and therefore we shall consider this case separately.

Lemma 6. Let $\left\{a_{j}\right\}$ be a basis of the parameter space $E$ and let us agree that for the orthonormalization coefficients $\lambda_{j}^{i}(x)$ from $(7), \lambda_{j}^{i}(x) \equiv 0$ for $i>j, i, j=$ $1,2, \ldots, k$, these coefficients satisfy (omitting for brevity the symbol $x$ )

$$
\left(\lambda_{q}^{j}\right)^{\prime} a_{r}+\sum_{i=1}^{q} \lambda_{q}^{i} \varkappa_{i}^{j} a_{r}=\left(\lambda_{r}^{j}\right)^{\prime} a_{q}+\sum_{i=1}^{r} \lambda_{r}^{i} \varkappa_{i}^{j} a_{q}, \quad\left\{\begin{array}{l}
1 \leqq j \leqq k \\
1 \leqq q<r \\
2 \leqq r \leqq k
\end{array}\right.
$$

and

$$
\sum_{i=1}^{q} \lambda_{q}^{i} \varkappa_{i}^{s, t} a_{r}=\sum_{i=1}^{r} \lambda_{r}^{i} \varkappa_{i}^{s, t} a_{q}, \quad\left\{\begin{array}{l}
(s, t) \in o_{2, k} \\
1 \leqq q<r \\
2 \leqq r \leqq k
\end{array}\right.
$$

In particular, for $j>r$ (31) contains no derivatives, and hence the number of differential equations in (31) is

$$
k\left(k^{2}-1\right) / 3 \text {, }
$$

the total number of equations in (31) and (32) together being

$$
\left(k+m_{2}\right) k(k-1) / 2,
$$

where $k=\operatorname{dim} M=\operatorname{dim} T_{y}^{1}(M), k+m_{2}=\operatorname{dim} T_{y}^{2}(M)$.

Proof. We have by (7) $y^{\prime} a_{q}=\sum_{i=1}^{k} \lambda_{q}^{i} e_{i}, q=1, \ldots, k$. Differentiate this with respect to $a_{r}(r \neq q)$ using (15):

$$
\begin{gathered}
y^{\prime \prime} a_{r} a_{q}=\sum_{j=1}^{k}\left(\lambda_{q}^{j}\right)^{\prime} a_{r} e_{j}+\sum_{i=1}^{k} \lambda_{q}^{i} e_{i}^{\prime} a_{r} \\
=\sum_{j=1}^{k}\left(\lambda_{q}^{j}\right)^{\prime} a_{r} e_{j}+\sum_{i=1}^{k} \lambda_{q}^{i}\left\{\sum_{j=1}^{k}\left(\varkappa_{i}^{j} a_{r}\right) e_{j}+\sum_{o_{2, k}}\left(\varkappa_{i}^{s, t} a_{r}\right) e_{s, t}\right\} \\
=\sum_{j=1}^{k}\left\{\left(\lambda_{q}^{j}\right)^{\prime} a_{r}+\sum_{i=1}^{k} \lambda_{q}^{i} \varkappa_{i}^{j} a_{r}\right\} e_{j}+\sum_{o_{2, k}}\left\{\sum_{i=1}^{k} \lambda_{q}^{i} \varkappa_{i}^{s, t} a_{r}\right\} e_{s, t} .
\end{gathered}
$$


One obtains (31) and (32) by interchanging $r$ and $q$ and equating the coefficients of the corresponding vectors.

The total number of equations in (31) and (32) is obtained by counting the number of vector equations $y^{\prime \prime} a_{q} a_{r}=y^{\prime \prime} a_{r} a_{q}(r \neq q)$ and the number of vectors from the accompanying orthonormal frame in these equations.

Consider now the general equation (12), taking into consideration the Remark after (14). Take an $a_{r}$ from the basis $\left\{a_{j}\right\}$ of $E$ and differentiate (12) with respect to it, use (15), and equate the result to a formula corresponding to $y^{(q+1)}$ in (12). Then we have

Lemma 7. Let $\bar{i}=\left(i_{1}, \ldots, i_{q}\right) \in O_{q, k}, \quad$ let $r \in\{1,2, \ldots, k\}$ and denote by $\bar{m}=\left(i_{1}, \ldots, r, \ldots, i_{q}\right) \in O_{q+1, k}$ the sequence corresponding to $\bar{i}$ after inserting $r$ into it in an appropriate place. Then for $q=1,2, \ldots, p,\left\{a_{j}\right\}$ being a basis of $E$,

$$
\begin{gathered}
\lambda_{m}^{j}=\left(\lambda_{i}^{j}\right)^{\prime} a_{r}+\sum_{l=1}^{k} \lambda_{i}^{l} \varkappa_{l}^{j} a_{r}+\sum_{l \in o_{2, k}} \lambda_{i}^{l} \varkappa_{l}^{j} a_{r}, \quad j=1, \ldots, k ; \\
\lambda_{\bar{m}}^{\bar{j}}=\left(\lambda_{i}^{\bar{j}}\right)^{\prime} a_{r}+\sum_{l \in o_{n-1, k}} \lambda_{i}^{l} \varkappa_{l}^{\bar{j}} a_{r}+\sum_{l \in o_{n, k}} \lambda_{i}^{\bar{l}} \varkappa_{l}^{\bar{j}} a_{r}+\sum_{l \in o_{n+1, k}} \lambda_{i}^{l} \varkappa_{l}^{\bar{j}} a_{r},
\end{gathered}
$$

where $\bar{j} \in o_{n, k}, n=2, \ldots, q-1$;

$$
\lambda_{\bar{m}}^{\bar{j}}=\left(\lambda_{i}^{\bar{j}}\right)^{\prime} a_{r}+\sum_{l \in o_{q-1, k}} \lambda_{i}^{\bar{l}} x_{l}^{\bar{j}} a_{r}+\sum_{l \in o_{q, k}} \lambda_{\bar{i}}^{l} x_{l}^{\bar{j}} a_{r}, \quad \bar{j} \in o_{q, k} ;
$$

and for $q<p$,

$$
\lambda_{\bar{m}}^{\bar{j}}=\sum_{l \in o_{q, k}} \lambda_{\bar{i}}^{l} \chi_{l}^{\bar{j}} a_{r}, \quad \bar{j} \in o_{q+1, k}, \quad \bar{j} \leqq \bar{m} .
$$

(For $q=1$, omit (35), (36) and the middle sum on the right side of (37), for $q=2$, omit (36).)

Here $\lambda_{\bar{m}}^{\bar{m}}(x)>0$ if $\bar{m} \in o_{q, k}, q=1, \ldots, p$.

Remark. Note that the equations (35)-(38) are recursive by nature, i.e. given the curvatures $\left\{\chi_{\bar{j}}^{\bar{i}}\right\}$ and the coefficients $\left\{\lambda_{\bar{r}}^{\bar{q}}\right\}$ where the lower index $\bar{r}$ belongs to $O_{q, k}$, the formulas (35)-(38) give the law for obtaining coefficients $\left\{\lambda_{\bar{t}}^{s}\right\}$ with the lower index $\bar{t}$ in the "higher" set $O_{q+1, k}$.

\section{Considerations on the embedding theorem}

Our version of the embedding theorem is the following

Theorem 1. Let us fix the integers $p, k \in N$ with $0<k<p$, and fix a complete ordering for the elements of the sets $O_{n, k}, n=1,2, \ldots, p$ such that $\bar{i}<\bar{j}$ whenever $\bar{i} \in O_{m, k}$ and $\bar{j} \in O_{n, k}$ with $m<n$. Let us also fix subsets $o_{n, k} \subset O_{n, k}$, with card $o_{n, k}=$ $m_{n}>0, n=1,2, \ldots, p ; m_{1}=k$. 
Let $E$ be a $k$-dimensional linear space over $R, U \subset E$ a connected region and $F$ a euclidean space with $\operatorname{dim} F=\sum_{i=1}^{p} m_{i}$.

Let there be given an orthonormal basis $\left\{e_{i}^{0}\right\}$ of $F$ and

$$
\sum_{i=1}^{p} m_{i}\left(m_{i}-1\right) / 2+\sum_{i=1}^{p-1} m_{i} m_{i+1}
$$

$C^{\infty}$ functions $x_{\bar{j}}^{\bar{i}}: U \rightarrow E^{*}$ (=the dual space of $E$ ), $\bar{j} \in o_{n, k}, \bar{i} \in o_{n, k}$ or $o_{n+1, k}$ for $n=1,2, \ldots, p-1$ and $\bar{i} \in o_{p, k}$ for $n=p ; \bar{j}<\bar{i}$, such that the following conditions are satisfied:

$\mathrm{i}^{\circ}$ The bilinear operator $B(x): E \times F \rightarrow F(x \in U)$ defined by means of its matrix (19) with respect to the basis $\left\{e_{i}^{0}\right\}$ satisfies the condition (25).

ii $^{\circ}$ The equations (31), (32), and (35)-(38) have a solution $\left\{\lambda_{\bar{j}}^{\bar{i}}(x) \mid \bar{i} \leqq j, \bar{j} \in o_{m, k}\right.$, $\left.\bar{j} \in O_{n, k}, 1 \leqq m \leqq n \leqq p\right\}$, satisfying

$$
\lambda_{i}^{i}(x)>0 \text { for all } x \in U \text { if } \quad \bar{i} \in o_{n, k},
$$

$n=1,2, \ldots, p$.

Then, given a point $y_{0} \in F$, there exists a $C^{\infty}$ surface $M: y=y(x), x \in U, M \subset F$, such that for $x \in U$ the functionals $\left\{x_{\tilde{j}}^{\bar{i}}(x)\right\}$ are its curvatures at $y(x) \in M, y\left(x_{0}\right)=y_{0}$, and the accompanying orthonormal frame of $M$ at $y_{0}$ is $\left\{e_{i}^{0}\right\}$. The dimension of the $n$-th tangent space $T_{\boldsymbol{y}}^{n}(M)$ is $k+m_{2}+\ldots+m_{n}, n=1,2, \ldots, p$.

Proof. The operator $B(x): E \times F \rightarrow F$ being defined as stipulated in $\mathrm{i}^{\circ}$, $B(x) a: F \rightarrow F$ is a linear alternating operator for each $a \in E, x \in U$, and satisfies the integrability condition (25) for the differential equation (24). Hence there exists a linear operator $T(x): F \rightarrow F$ for each $x \in U$.

The operator $T(x)$ is orthogonal, as can be seen by differentiation of $T(x) T(x)^{*}$ with respect to an arbitrary $a \in E(a \neq 0)$ and use of (24) together with (23). Then $\left(T(x) T(x)^{*}\right)^{\prime} a=0$, and by the fundamental theorem of integral calculus (see [5, p. 81]) $T(x) T(x)^{*}=$ constant $=T\left(x_{0}\right) T\left(x_{0}\right)^{*}=I$.

Let $e_{i}^{0}=e_{i}\left(x_{0}\right), i=1,2, \ldots, \operatorname{dim} F$. Then the equation

$$
T(x) e_{\bar{j}}\left(x_{0}\right)=e_{\bar{j}}(x), \quad \bar{j} \in o_{n, k}, \quad n=1, \ldots, p, \quad x \in U
$$

defines an orthonormal basis of $F$ for each $x \in U$ (here $\bar{j}$ is the element on the $i$-th place in the sequence of the elements of $\left.o_{n, k}, n=1,2, \ldots, p, i=1,2, \ldots, \operatorname{dim} F\right)$.

According to the hypothesis there exists a basis $\left\{a_{j}\right\}$ of $E$ such that the equations (31) and (32) from Lemma 6 have a solution. This makes it possible for us to construct a system of $k$-dimensional subspaces in $F$ (i.e. the first tangent spaces of our surface) as follows: a solution being $\left\{\lambda_{j}^{i}(x) \mid 1 \leqq i \leqq j \leqq k, x \in U\right\}$, define vectors $z_{j}(x) \in F, j=1,2, \ldots, k$ by means of $k$ first vectors from (40) by

$$
z_{j}(x)=\sum_{i=1}^{j} \lambda_{j}^{i}(x) e_{i}(x), \quad x \in U, \quad j=1,2, \ldots, k
$$


Since $o_{1, k}=\{1,2, \ldots, k\}$, (39) gives $\lambda_{j}^{j}(x)>0$ for $j=1,2, \ldots, k$. Hence the vectors (41) are linearly independent spanning a $k$-dimensional linear subspace

$$
T_{y}^{1}(M)=\operatorname{sp}\left\{z_{1}(x), \ldots, z_{k}(x)\right\} \subset F, \quad x \in U .
$$

Let us now define a linear bijection $Z(x): E \rightarrow T_{y}^{1}(M)$ by

$$
Z(x) a_{i}=z_{i}(x), \quad i=1,2, \ldots, k, \quad x \in U .
$$

We want $T_{y}^{1}(M)$ to be the first tangent space of a surface $M$. Hence by the definition (5) for $n=1$, the linear mapping $Z(x)$ must be the derivative of a differentiable mapping $y: U \rightarrow F$, i.e. we have the differential equation

$$
y^{\prime}(x) a=Z(x) a, \quad a \in E, \quad x \in U \subset E .
$$

The integrability condition for (44) is (by $[5$, p. 141])

$$
\operatorname{rot} Z(x) \equiv 0 \text {, }
$$

where $\operatorname{rot} Z(x)$ is the bilinear mapping $E \times E \rightarrow F$ defined by

$$
[\operatorname{rot} Z(x)](a, b)=(1 / 2)\left[Z^{\prime}(x) a b-Z^{\prime}(x) b a\right], \quad a, b \in E, \quad x \in U .
$$

It is enough that (45) holds for the basis $\left\{a_{i}\right\}$ of $E$, i.e. $Z^{\prime}(x) a_{i} a_{j}=Z^{\prime}(x) a_{j} a_{i}$, $i, j=1,2, \ldots, k, i \neq j$, or, by (43), $z_{i}^{\prime}(x) a_{j}=z_{j}^{\prime}(x) a_{i}, i, j=1, \ldots, k, i \neq j$. This, however, leads us by (41) back to Lemma 6 , the equations of which are satisfied by the hypothesis $\mathrm{ii}^{\circ}$.

Hence (45) is satisfied and the function (see [5, p. 120] line integral)

$$
y=y(x)=y_{0}+\int_{x_{0}}^{x} Z(x) d x \quad\left(x, x_{0} \in U\right)
$$

is defined, representing a $C^{\infty}$ surface $M$ in $F$.

It is now necessary to check that this surface actually possesses the properties of the Theorem. One can perform the orthogonalization procedure described in the Sections 2 and 3, and, since (39) completely describes the linear dependence and independence of the vectors in question, the dependence structure in the tangent spaces coincides with the one given in the Theorem; in particular,

$$
\operatorname{dim} T_{y}^{n}(M)=k+m_{2}+\ldots+m_{n}, \quad n=1, \ldots, p .
$$

Moreover, the accompanying orthonormal frame of $M$ is the same as given in (40), since the coefficients $\left\{\lambda_{\bar{j}}^{\bar{i}}\right\}$ of the orthonormalization procedure are obtained from the recurrence relations (35)-(38) for a given solution $\left\{\lambda_{j}^{i}\right\}$ of (31) and (32). This implies at once that the curvatures of $M$ coincide with the given functions $\left\{x_{\bar{j}}^{\bar{i}}(x)\right\}, x \in U$, the rest of the proof being clear by the initial conditions.

Remark 1 . We have considered $C^{\infty}$ surfaces. For weaker differentiability conditions, see [6]. 
Remark 2. As regards uniqueness, we note that the equations (31) and (32) can have several solutions, each of which gives rise to a new set of coefficients $\left\{\lambda_{j}^{\bar{i}}\right\}$ recursively. With suitable additional requirements concerning the parametrization (e.g. given the $g_{i j}(x)$ ), one could also achieve uniqueness.

Remark 3. Note that the condition $\mathrm{i}^{\circ}$ with only that part of $\mathrm{ii}^{\circ}$ which refers to the tangent space $T_{y}^{1}(M)$ gives the embedding theorem of [6, p. 26].

\section{Degeneration of the surface}

We have assumed in the above discussion that a basis can be found for the embedding space $F$ from the first $p$ derivatives of the embedding function $y: U \rightarrow F$ differentiated with respect to a basis $\left\{a_{j}\right\}$ of the parameter space $E$.

We say that a surface $M$ is degenerate if there exists an integer $p$ such that for all $i, j: 1 \leqq i<j \leqq p, T_{y}^{i}(M) \subseteq T_{y}^{j}(M)$, but

$$
T_{y}^{p+1}(M)=T_{y}^{p}(M) \subsetneq F .
$$

The integer $p$ is then called the degree of degeneration of the surface $M$ and $M$ is degenerate to the degree $p$.

Theorem 2. Suppose a surface $M$ is degenerate to the degree $p$. Then it lies within the affine subspace

$$
y\left(x_{0}\right)+T_{y_{0}}^{p}(M) \quad\left(y_{0}=y\left(x_{0}\right) \in M, x_{0} \in U \text { fixed }\right)
$$

of the embedding space $F$.

Proof. We can construct an orthonormal basis for $T_{y}^{p}(M)$ by using Schmidt's orthonormalization procedure. For a fixed $x_{0} \in U \subset E$, we can define the operator $T(x): T_{y_{0}}^{p} \rightarrow F$ by means of the formula (21). $T(x)$ is now an isometric operator $T(x): T_{y_{0}}^{p}(M) \rightarrow T_{y}^{p}(M)$, where $y=y(x)$ varies as a function of $x \in U$.

Now

$$
P(x)=T(x) T(x)^{*}
$$

is an orthogonal projection $P(x): F \rightarrow T_{y_{0}}^{p}(M)$, since it satisfies $P(x)=P(x)^{*}=$ $P(x)^{2}$ by virtue of the fact that $T(x)^{*} T(x)=$ the identity of $T_{y_{0}}^{p}(M)$.

The operator $A(x) a: T_{y}^{p}(M) \rightarrow T_{y}^{p}(M)$ from (16) and $B(x) a: T_{y_{0}}^{p} \rightarrow T_{y_{0}}^{p}$ can be defined, and (23) is satisfied.

Moreover, the differential equation (24) holds with the initial condition $T\left(x_{0}\right)=$ $I=$ the identity of $T_{y_{0}}^{p}(M)$.

Differentiation of (50) with respect to an $a \in E(a \neq 0)$, by use of (24) and the skew-symmetry of $B(x) a$, yields $P^{\prime}(x) a=0$. Now the fundamental theorem of integral calculus (see $[5, \mathrm{p} .81]$ ) implies that $P(x)=$ constant $=P\left(x_{0}\right)$. 
Since $y^{\prime}(z) d z \in T_{y}^{1}(M) \subset T_{y}^{p}(M)$ for $z \in U, d z \in E$, we have $y^{\prime}(z) d z=P(z) y^{\prime}(z) d z=$ $P\left(x_{0}\right) y^{\prime}(z) d z$, and

$$
\begin{aligned}
y(x)-y\left(x_{0}\right) & =\int_{x_{0}}^{x} y^{\prime}(z) d z=\int_{x_{0}}^{x} P\left(x_{0}\right) y^{\prime}(z) d z= \\
& =P\left(x_{0}\right) \int_{x_{0}}^{x} y^{\prime}(z) d z=P\left(x_{0}\right)\left(y(x)-y\left(x_{0}\right)\right),
\end{aligned}
$$

showing that $y(x)-y\left(x_{0}\right) \in T_{y}^{p}\left(x_{0}\right)$ for all $x \in U$.

\section{Particular cases}

8.1. Hypersurfaces. A hypersurface is a $k$-dimensional non-degenerate surface in a $(k+1)$-dimensional euclidean space $F$. By Theorem 2, there exists a pair $(s, t) \in O_{2, k}$ such that $y^{\prime \prime}(x) a_{s} a_{t}$ is linearly independent of $\left\{y^{\prime}(x) a_{i}\right\}_{1}^{k}$ for all $x \in U \subset E$. Hence a hypersurface is always of type $(k, 1)$.

In the Frenet matrix (19) $A_{11}$ is an antisymmetric $k \times k$-matrix and $A_{21}$ is a $1 \times k$-matrix, i.e. a row vector, $A_{22}$ being the scalar 0 .

We are examining a connection between our Theorem 1 and the embedding theorem for hypersurfaces $[4$, p. $129 ; 5$, p. 226].

The Frenet matrix of a hypersurface takes the form

$$
[A(x) a]=\left(\begin{array}{ccccc}
0 & -\chi_{1}^{2}(x) a & \ldots & -\chi_{1}^{k}(x) a & -\chi_{1}^{s, t}(x) a \\
\chi_{1}^{2}(x) a & 0 & -\chi_{2}^{k}(x) a & -\chi_{2}^{s, t}(x) a \\
\vdots & \vdots & & & \vdots \\
\chi_{1}^{k}(x) a & \chi_{2}^{k}(x) a & \ldots & 0 & -\chi_{k}^{s, t}(x) a \\
\chi_{1}^{s, t}(x) a & \chi_{2}^{s, t}(x) a & \ldots & \chi_{k}^{s, t}(x) a & 0
\end{array}\right),
$$

the accompanying orthonormal frame being $\left\{e_{i}(x)\right\}_{1}^{k} \cup\left\{e_{s, t}(x)\right\}$.

Given the first fundamental form $G(x) a b$ and fixing a basis $\left\{a_{j}\right\}_{1}^{k}$ of $E$, one obtains the orthonormalization coefficients $\left\{\lambda_{j}^{i}\right\}$ from (11). Letting

$$
\left\{x_{j}^{i}(x) a_{q} \mid 1 \leqq j<i \leqq k, q=1, \ldots, k\right\}
$$

be the unknowns, one has in (31) a system of linear equations with an equal number $k^{2}(k-1) / 2$ of equations and unknowns. Since a linear functional $\chi_{j}^{i}(x): E \rightarrow \boldsymbol{R}$ is uniquely determined by means of its values for the basis vectors $\left\{a_{j}\right\}$ of $E$, one gets from (31) the curvatures $\left\{\chi_{j}^{i}(x) \mid 1 \leqq j<i \leqq k\right\}$ in terms of $g_{i j}(x)=G(x) a_{i} a_{j}, 1 \leqq i \leqq$ $j \leqq k$.

Since the practical computation appears rather tedious in the general case, we give the formulas for $k=2$ only. In this case (31) yields only two equations: $q=1$, 
$r=2$ and $j=1$ or $j=2$. Bearing in mind that $\lambda_{1}^{2}=\chi_{1}^{1}=\chi_{2}^{2}=0$ and $\chi_{2}^{1} a_{1}=-\chi_{1}^{2} a_{1}$, we obtain from (31), omitting the symbol $x$,

$$
\left\{\begin{array}{l}
x_{1}^{2} a_{1}=\left[\left(\lambda_{2}^{1}\right)^{\prime} a_{1}-\left(\lambda_{1}^{1}\right)^{\prime} a_{2}\right]\left(\lambda_{2}^{2}\right)^{-1} \\
\varkappa_{1}^{2} a_{2}=\left[\left(\lambda_{2}^{2}\right)^{\prime} a_{1}+\lambda_{2}^{1} \varkappa_{1}^{2} a_{1}\right]\left(\lambda_{1}^{1}\right)^{-1}
\end{array}\right.
$$

Now we obtain from (11)

$$
\lambda_{1}^{1}=g_{11}^{1 / 2}, \quad \lambda_{2}^{1}=g_{12} g_{11}^{-1 / 2}, \quad \lambda_{2}^{2}=\left(g_{11} g_{22}-g_{12}^{2}\right)^{1 / 2} g_{11}^{-1 / 2} .
$$

Inserting (54) into (53) yields

and

$$
\varkappa_{1}^{2} a_{1}=(1 / 2)\left\{2 g_{11} g_{12}^{\prime} a_{1}-g_{12} g_{11}^{\prime} a_{1}-g_{11} g_{11}^{\prime} a_{2}\right\} g_{11}^{-1}\left(g_{11} g_{22}-g_{12}^{2}\right)^{-1 / 2}
$$

$$
x_{1}^{2} a_{2}=(1 / 2)\left\{g_{11} g_{22}^{\prime} a_{1}-g_{12} g_{11}^{\prime} a_{2}\right\} g_{11}^{-1}\left(g_{11} g_{22}-g_{12}^{2}\right)^{-1 / 2} \text {. }
$$

Moreover, we have the equations (32), the number of which is $k(k-1) / 2$ in the case of a hypersurface. This is not enough to solve all the unknowns

$$
\left\{x_{i}^{s_{i} t} a_{j} \mid i, j=1, \ldots, k\right\},
$$

the number of which is $k^{2}$.

Now, the second fundamental form $L(x): E \times E \rightarrow \boldsymbol{R}(x \in U)$ is defined by (see [5, p. 196])

$$
L(x) a b=\left(y^{\prime \prime}(x) a b \mid e_{s, t}(x)\right) .
$$

This being symmetrical with respect to $a$ and $b \in E$, it can be given by means of the $k(k+1) / 2$ quantities

Since by (12)

$$
l_{i j}(x)=L(x) a_{i} a_{j}, \quad 1 \leqq i \leqq j \leqq k .
$$

$$
y^{\prime \prime}(x) a_{i} a_{j}=\sum_{s=1}^{k} \lambda_{i, j}^{s} e_{s}+\lambda_{i, j}^{s, t} e_{s, t}
$$

(which, incidentally, is one form of Gauss's derivative formula; see [5, p. 196]), we have

$$
l_{i j}(x)=\lambda_{i, j}^{s, t}(x) \quad 1 \leqq i \leqq j \leqq k .
$$

If we use Lemma 7 for $q=1$ (or, equivalently, the equation used in the proof of Lemma 6), (38) gives

$$
\lambda_{i, j}^{s, t}=\sum_{l=1}^{k} \lambda_{i}^{l} \varkappa_{l}^{s, t} a_{j} \quad 1 \leqq i \leqq j \leqq k,
$$

and now (56) and (57) together with (11) yield the missing $k(k+1) / 2$ equations for the unknowns (55). Now we have $k^{2}$ equations for these $k^{2}$ unknowns, and solving them we obtain the curvatures by means of the first and second fundamental forms. Hence we get from Theorem 1 as a corollary the embedding theorem for hypersurfaces $[4$, p. $129 ; 5$, p. 226$]$. 
In the particular case of $k=2$, the equations needed in addition to those following (54) are

and

$$
\begin{aligned}
& \varkappa_{\mathrm{i}}^{s^{5} t} a_{1}=l_{11}\left(g_{11}\right)^{-1 / 2}, \\
& x_{1}^{s, t} a_{2}=l_{12}\left(g_{11}\right)^{-1 / 2} \text {, } \\
& \varkappa_{2}^{\mathrm{s}, t} a_{1}=\left(g_{11} l_{12}-g_{12} l_{11}\right) g_{11}^{-1 / 2}\left(g_{11} g_{22}-g_{12}^{2}\right)^{-1 / 2} \text {, }
\end{aligned}
$$$$
\varkappa_{2}^{s, t} a_{2}=\left(g_{11} l_{22}-g_{12} l_{12}\right) g_{11}^{-1 / 2}\left(g_{11} g_{22}-g_{12}^{2}\right)^{-1 / 2} .
$$

8.2. On surfaces with constant curvatures. Consider the problem of finding a non-degenerate surface with given constant curvatures, i.e. with a given Frenet matrix of constant functionals.

Fixing an orthonormal basis $\left(e_{i}^{0}\right)_{1}^{m}$ of the embedding space $F$, one obtains the operator $B a: F \rightarrow F, a \in E$.

The integrability condition (25) for the differential equation (24) reduces to

$$
B a B b=B b B a, \quad a, b \in E .
$$

Hence $\{B x \mid x \in E\}$ is a system of intercommuting linear skew operators, i.e. an Abelian system of skew operators (can also be considered as a commutative semisimple set [1, p. 432]), depending linearly on the parameter $x \in E$.

Lemma 8. Given an Abelian system $\{B x \mid x \in E\}$ of skew operators $F \rightarrow F$, depending linearly on the parameter $x \in E$, there exists an orthonormal basis $\left(h_{j}\right)_{1}^{m}$ of $F$, an integer $q$, and nonzero linear functionals $\varrho_{i}: E \rightarrow \boldsymbol{R}(i=1, \ldots, q)$ such that

$$
\left\{\begin{array}{l}
B x h_{2 i-1}=\varrho_{i}(x) h_{2 i} \\
B x h_{2 i}=-\varrho_{i}(x) h_{2 i-1}
\end{array} \quad i=1, \ldots, q, \quad x \in E,\right.
$$

and

$$
B x h_{i}=0 \text { for } i=2 q+1, \ldots, m=\operatorname{dim} F .
$$

Proof. Denote by $G=F+i F$ the complexification of $F$ [3, pp. 150-152]. Then $G$ possesses a Hermitian form $(\mid)^{c}: G \times G \rightarrow C$ such that its restriction to $F$ coincides with the euclidean scalar product of $F$. Hence we can consider $G$ as a unitary space.

Also an $\boldsymbol{R}$-linear operator $A: F \rightarrow F$ can be extended to $G$ in such a way that its algebraic properties are preserved, e.g. if $A$ is skew in $F$, then its extension $A^{c}: G \rightarrow G$ is skew-Hermitian.

Fix a conjugation in $G[1$, p. 331, 334] such that $F$ is the set of real vectors with respect to this conjugation.

Let $\left\{(B x)^{c} \mid x \in E\right\}$ be the skew-Hermitian Abelian system in $L(G ; G)$ corresponding to $\{B x \mid x \in E\} \subset L(F ; F)$. For each $x \in E,(B x)^{c}$ is a real operator with respect to the conjugation (i.e. it commutes with the conjugation). In a unitary space there is no essential difference between selfadjoint and skew mappings; hence the nonzero proper values of $(B x)^{c}$ are located symmetrically with respect to zero on the imaginary 
axis. Given an $x_{0} \in E, G$ can be decomposed as an orthogonal direct sum of the eigensubspaces of $\left(B x_{0}\right)^{c}$, corresponding to distinct eigenvalues, the dimension of an eigensubspace being equal to the multiplicity of the corresponding eigenvalue [3, p. 154].

After these prerequisites we start proving our Lemma.

First we prove that $G$ can be decomposed into mutually orthogonal subspaces $G_{j}(j=1, \ldots, r)$ :

$$
G=G_{1} \oplus G_{2} \oplus \ldots \oplus G_{r} \quad\left(\operatorname{dim} G_{j} \geqq 1 \text { for all } j\right)
$$

such that for all $j$, every element $u \in G_{j}$ is an eigenvector of every $(B x)^{c}, x \in E$.

The decomposition (61) can be established as follows. Take $x_{1} \in E, x_{1} \neq 0$. The operator $\left(B x_{1}\right)^{c}$ divides the space $G$ into an orthogonal direct sum of its eigensubspaces $G_{j}^{(1)}, \operatorname{dim} G_{j}^{(1)} \geqq 1, j=1, \ldots, r_{1}$ :

$$
G=G_{1}^{(1)} \oplus G_{2}^{(1)} \oplus \ldots \oplus G_{r_{1}}^{(1)} .
$$

As regards a $G_{j}^{(1)}$ from (62), there are two possibilities:

$i^{\circ}$ every element $u \in G_{j}^{(1)}$ is an eigenvector of every $(B x)^{c}, x \in E$, and

ii $^{\circ}$ there exist an $x_{2} \in E$ and a $u \in G_{j}^{(1)}$ such that $u$ is not an eigenvector of $\left(B x_{2}\right)^{c}$.

If $\mathrm{i}^{\circ}$ holds, then $G_{j}^{(1)}$ is acceptable to (61). Suppose that ii $^{\circ}$ holds. Then necessarily $x_{1}$ and $x_{2}$ are linearly independent and $\operatorname{dim} G_{j}^{(1)} \geqq 2$.

Since $\left(B x_{1}\right)^{c}$ and $\left(B x_{2}\right)^{c}$ commute, $G_{j}^{(1)}$ is a stable subspace of $\left(B x_{2}\right)^{c}$; in fact, for $u \in G_{j}^{(1)},\left(B x_{1}\right)^{c}\left(B x_{2}\right)^{c} u=\left(B x_{2}\right)^{c}\left(B x_{1}\right)^{c} u=\lambda_{j}\left(B x_{2}\right)^{c} u$, where $\lambda_{j}$ is the eigenvalue of $\left(B x_{1}\right)^{c}$ corresponding to $G_{j}^{(1)}$.

Now the restriction of $\left(B x_{2}\right)^{c}$ into $G_{j}^{(1)}$ is a skew-Hermitian mapping $G_{j}^{(1)} \rightarrow G_{j}^{(1)}$, and this space can be decomposed according to the eigensubspaces of that mapping.

Accordingly, the decomposition (62) of $G$ can be refined with $\left(B x_{2}\right)^{c}$ orthogonally into

$$
G=G_{1}^{(2)} \oplus \ldots \oplus G_{r_{2}}^{(2)}, \quad \operatorname{dim} G_{j}^{(2)} \geqq 1, \quad j=1,2, \ldots, r_{2},
$$

$\left(r_{2}>r_{1}\right)$ such that every element $u \in G_{j}^{(2)}$ is an eigenvector of both $\left(B x_{1}\right)^{c}$ and $\left(B x_{2}\right)^{c}$.

Now one can check the possibilities $i^{\circ}$ and ii $^{\circ}$ for the decomposition (63).

However, this refinement cannot be continued infinitely, because we are dealing with finite-dimensional spaces. After a finite number of steps we obtain the decomposition (61).

Then, given any $u \in G_{j}$, there exists for each $x \in E$ a real number $\varrho(x)$ such that

$$
(B x)^{c} u=i \varrho(x) u
$$

(since $(B x)^{c}$ is skew-Hermitian) and the dependence of $\varrho$ on $x$ is linear.

Fix now an orthonormal basis for each $G_{j}(j=1, \ldots, r)$ from (61). The aggregate of these bases is then an orthonormal basis of $G$, denoted by $\left\{u_{j}\right\}_{1}^{m}\left(m=\operatorname{dim}_{C} G=\operatorname{dim}_{R} F\right)$.

Then, according to (64), we have nonzero linear functionals $\varrho_{j}: E \rightarrow R, j=1, \ldots, q$ such that

$$
(B x)^{c} u_{j}=i \varrho_{j}(x) u_{j}, \quad j=1, \ldots, q .
$$


Letting $\bar{u}_{j}$ be the conjugate vector of $u_{j}$, we have in view of the reality of $(B x)^{c}$,

$$
(B x)^{c} \bar{u}_{j}=-i \varrho_{j}(x) \bar{u}_{j} . \quad j=1, \ldots, q,
$$

$\varrho_{j}(x)$ depending linearly on $x \in E, j=1, \ldots, q$.

Since $\left(u_{j} \mid \bar{u}_{j}\right)^{c}=0$, we have $\left|u_{j}+\bar{u}_{j}\right|=\left|u_{j}-\bar{u}_{j}\right|=\sqrt{2}, j=1,2, \ldots, q$. Letting

$$
h_{2 j-1}=\frac{1}{\sqrt{2}}\left(u_{j}+\bar{u}_{j}\right) \quad \text { and } \quad h_{2 j}=\frac{i}{\sqrt{2}}\left(u_{j}-\bar{u}_{j}\right), \quad j=1,2, \ldots, q,
$$

we obtain the orthonormal real unit vectors satisfying (59).

(60) is obtained in a natural manner by means of a real orthonormal basis of $\bigcap_{x \in E} \operatorname{Ker}(B x)^{c}$.

Remark. Note that (59) holds for an arbitrary orthonormal set $\left\{k_{j}\right\}_{1}^{2 q}$ such that $k_{2 i-1}$ and $k_{2 i}$ belong to the two-dimensional (over $R$ ) linear space $\operatorname{sp}\left\{h_{2 i-1}, h_{2 i}\right\} \subset F$, $i=1, \ldots, q$.

Now $\left(e_{i}^{0}\right)_{1}^{k}$ span the tangent space $T_{y_{0}}^{1}(M)$ at $y_{0}=y\left(x_{0}\right)$. Projecting $e_{i}^{0}$ on sp $\left\{k_{2 j-1}, k_{2 j}\right\}(j=1, \ldots, q, i=1, \ldots, k)$, where $\left(k_{j}\right)_{1}^{2 q}$ is an orthonormal system satisfying (59), and denoting the projection by $\alpha_{i}^{j} h_{2 j} \quad\left(\left|h_{2 j}\right|=1\right)$, we have

$$
e_{i}^{0}=\sum_{j=1}^{q} \alpha_{i}^{j} h_{2 j}+\sum_{j=2 q+1}^{m} \alpha_{i}^{j} h_{j}, \quad i=1, \ldots, q .
$$

Then, after some calculations involving the exponential $T(x)=\exp (B x)$ (see [5, p. 166]) one obtains, letting $h_{2 j-1} \in \operatorname{sp}\left\{k_{2 j-1}, k_{2 j}\right\}$ be a unit vector orthogonal to $h_{2 j}, j=1, \ldots, q$,

$$
\begin{aligned}
e_{i}(x)=T(x) e_{i}^{0} & =-\sum_{n=1}^{q} \alpha_{i}^{n}\left\{\sin \varrho_{n}(x) h_{2 n-1}-\cos \varrho_{n}(x) h_{2 n}\right\} \\
& +\sum_{n=2 q+1}^{m} \alpha_{i}^{n} h_{n}, \quad x \in U, \quad i=1, \ldots, k .
\end{aligned}
$$

Note that here the linear functionals $\left\{\varrho_{n}\right\}$ should be pairwise different elements of $E^{*}$, and $m-2 q \leqq k$, or else the surface will be degenerate. Note also that the expression (67) bears some resemblance to the expression for curves with constant curvatures [5, p. 192].

The vectors $\left(e_{i}(x)\right)_{1}^{k}$ are a result of the orthogonalization of $\left(y^{\prime}(x) a_{i}\right)$ in $T_{y}^{1}(M)$, where the coefficients $\left(\lambda_{j}^{i}\right)$ satisfy (31) and (32). These equations contain too much arbitrariness due to the lack of a canonical parametrization (e.g. arc length for curves).

Let us impose some extra conditions for more detailed results. We want a surface with constant curvatures and with an orthogonal parametrization. We take

$$
y^{\prime}(x) a_{i}=\lambda_{i}^{i} e_{i}(x), \quad i=1, \ldots, k,
$$

$\lambda_{i}^{i}$ being constant, $i=1, \ldots, k$ ( $g_{i j}$ being constants, too).

Let $x=\sum_{i=1}^{k} x_{i} a_{i}$ and $\varrho_{n}(x)=\sum_{l=1}^{k} \beta_{n}^{l} x_{l}, x_{1}, \ldots, x_{k} \in \boldsymbol{R}$. In order that (67) should represent, up to certain constant factors, the derivatives of an embedding 
$y=y(x)$, it is sufficient that the factors $\lambda_{i}^{i}>0$ should satisfy for all $x \in U$

$$
\lambda_{i}^{i} \frac{\partial e_{i}}{\partial x_{j}}(x)=\lambda_{j}^{j} \frac{\partial e_{j}}{\partial x_{i}}(x), \quad 1 \leqq i<j \leqq k .
$$

This leads to the $q k(k-1) / 2$ equations

$$
\lambda_{i}^{i} \alpha_{i}^{n} \beta_{n}^{j}=\lambda_{j}^{j} \alpha_{j}^{n} \beta_{n}^{i}, \quad n=1,2, \ldots, q, \quad 1 \leqq i<j \leqq k
$$

for the $k$ unknowns $\left(\lambda_{i}^{i}\right)_{1}^{k}, \lambda_{i}^{i}>0, i=1, \ldots, k$.

If these equations are satisfied, the embedding

$$
y=y(x)=\lambda_{i}^{i}\left\{\sum_{n=1}^{q} \frac{\alpha_{i}^{n}}{\beta_{n}^{i}}\left[\cos \varrho_{n}(x) h_{2 n-1}+\sin \varrho_{n}(x) h_{2 n}\right]\right\}+\sum_{n=2 q+1}^{m} \sum_{i=1}^{k} \alpha_{i}^{n} x_{i} h_{n}, x \in U
$$

gives such a surface. However, this does not yield a very wide class of surfaces, because the number of equations in (69) increases rapidly with $k$.

Examples of these surfaces are Cartesian products of curves with constant curvatures, e.g. the ordinary cylinder in $\boldsymbol{R}^{3}$, the Cartesian product of a circle with itself in $\boldsymbol{R}^{4}$ (being of type $(2,2)$ ), and the Cartesian product of the circular helix and the straight line:

$$
M=\left\{(a \cos b u, a \sin b u, c u, d v) \mid(u, v) \in \boldsymbol{R}^{2}\right\}
$$

( $a, b, c, d \in \boldsymbol{R}, a, b, c, d \neq 0)$, this surface in $\boldsymbol{R}^{4}$ being of type $(2,1,1)$.

\section{References}

[1] Greub, W. H.: Linear algebra. - 4th ed., Springer-Verlag, Berlin-Heidelberg-New York, 1975.

[2] Guggenheimer, Heinrich W.: Differential geometry. - McGraw-Hill Book Company, Inc., New York-San Francisco-Toronto-London, 1963.

[3] Halmos, Paul R.: Finite-dimensional vector spaces. - Springer-Verlag, Berlin-HeidelbergNew York, 1974.

[4] Hicks, Noel J.: Notes on differential geometry. - D. Van Nostrand Company, Inc., Princeton, New Jersey-Toronto-New York-London, 1965.

[5] Nevanlinna, F. and R.: Absolute Analysis. - Springer-Verlag, Berlin-Heidelberg-New York, 1973.

[6] Rikkonen, HaRri: Zur Einbettungstheorie. - Ann. Acad. Sci. Fenn. Ser. A I 300, 1961, 1-28.

[7] SPIVAK, M.: A comprehensive introduction to differential geometry. Volumes 4 and 5. - Publish or Perish, Inc., Boston, Mass. 02108, 1975.

University of Oulu

Department of Mathematics

SF-90570 Oulu 57

Finland

Received 5 May 1978

Revision received 26 June 1978 